\title{
The flow rate sensitivity to voltage across four electrospray modes
}

\author{
C. N. Ryan, ${ }^{1, a)}$ K. L. Smith, ${ }^{2, b)}$ and J. P. W. Stark ${ }^{1}$ \\ ${ }^{1}$ School of Engineering and Materials Science, Queen Mary University of London, London E1 4NS, \\ United Kingdom \\ ${ }^{2}$ School of Mechanical, Aerospace and Civil Engineering, The University of Manchester, \\ Manchester M13 9PL, United Kingdom
}

(Received 10 September 2013; accepted 11 February 2014; published online 25 February 2014)

The influence of potential difference on the emitted flow rate across four modes of electrospray is described for an unrestricted electrospray system. The modes are those most commonly occurring; enhanced dripping, pulsation, cone-jet, and multi-jet. It is demonstrated that within three of these modes, the effect of voltage on flow rate is generally linear, with similar magnitude of gradient across all. The effect is demonstrated to be calculable across these three modes. This finding highlights that in the absence of any flow control mechanism, the influence of electrostatic pressure in driving the flow is the key process in voltage-driven electrospray. (C) 2014 AIP Publishing LLC. [http://dx.doi.org/10.1063/1.4866670]

Electrospray is an electrohydrodynamic (EHD) process whereby the application of an electric field at the free surface of a conductive fluid results in the deformation of the liquid meniscus, resulting in the emission of drops of fluid. It is a commonly applied technique, especially within electrospray mass spectrometry ${ }^{1}$ (ESMS), EHD printing, ${ }^{2,3}$ spacecraft electric micro propulsion, ${ }^{4,5}$ and electrospinning. ${ }^{6}$

At lower electric fields (applied voltages) an "enhanced dripping" mode occurs, where the drops remain approximately the size of the emitter, but the electric field acts to increase the frequency of individually emitted droplets whilst decreasing their size. ${ }^{7,8}$

At higher electric field strength, intermittent cone like apices are formed; defined as the pulsation cone-jet regime. ${ }^{9,10}$ The charge accumulates on the liquid surface, with a resulting tangential stress leading to a cyclical emission of charged droplets substantially smaller than the emitter diameter. ${ }^{11}$

Further increases in the electric field intensity result in the formation of a stable Taylor cone. ${ }^{12}$ At the apex of the cone the equipotential nature, and thus the conical structure, breaks down and a thin jet is formed, breaking up to produce a quasi-monodisperse spray. This has been defined as conejet mode electrospray. At even higher field intensity, the cone-jet bifurcates with a jet emanating from each of the two cones, described as the multi-jet regime. Additional increases in electric field result in more cone-jet protrusions, ${ }^{9,13,14}$ each with identical flow rate-current characteristics as in the single cone-jet structure. ${ }^{15,16}$

The most commonly employed electrospray regime is cone-jet mode electrospray, although pulsation electrospray has found some uses in both ESMS ${ }^{17,18}$ and electrostatic printing. ${ }^{19}$ Multi-jet electrospray has few applications due to the small stable operating window of the regime. ${ }^{16}$

Which mode occurs is dependent upon the applied potential difference between the emitter and extractor. However, the detailed influence of the applied voltage or

\footnotetext{
${ }^{\text {a)} E l e c t r o n i c ~ m a i l: ~ c . n . r y a n @ q m u l . a c . u k . ~}$

b)Electronic mail: kate.smith@manchester.ac.uk.
}

electric field on the spray properties remains undetermined. One uncertainty is the relational dependence of emitted flow rate upon applied potential difference when the electrospray is unconstrained by a device such as a syringe pump. Within cone-jet mode, the dependence of flow rate upon the applied voltage has generally been excluded from theoretical analysis on the basis that the effect was assumed to be of marginal significance. ${ }^{20}$ However, under certain conditions within cone-jet mode electrospray the applied voltage may significantly influence the emitted flow rate. ${ }^{21-25}$ These studies revealed that the flow rate increases linearly with the applied potential difference, and that a flow rate increase of up to $45 \%$ can be achieved. ${ }^{22}$ Many geometric parameters were identified as controlling the flow rate-to-voltage sensitivity, ${ }^{25}$ captured using an analytical electrostatic model, revealing the role of electric pressure in this process.

This article extends the flow rate-to-voltage (termed the $Q(V)$ ) relationship) investigation to include the relationship between the emitted flow rate and applied voltage in four electrospray modes; enhanced dripping, pulsation, cone-jet, and multi-jet.

The results presented here were obtained using electrospray methods described previously. ${ }^{22,24}$ The fluid was supplied from a gravity-fed reservoir to a non-tapered stainless steel emitter. In the fluid line between reservoir and emitter two pressure transducers acted as a highly accurate flow meter with a resolution of a fraction of a nano-liter per second. ${ }^{21}$ The underlying principle of the high accuracy flow meter is based upon the use of the Hagen-Poiseuille equation. ${ }^{13,22}$ The applied potential difference was supplied by a high voltage unit connected between the extractor electrode, having an aperture through which the electrospray droplets were accelerated, and a capillary emitter held at virtual ground potential.

The results were obtained using three liquids. Two organic solvents were tested; propylene carbonate (PC, used for the majority of the experiments), and ethylene glycol (EG), both solutions being doped with NaI to obtain conductivities of $\sigma=0.003$ and $0.23 \mathrm{~S} / \mathrm{m}$, respectively. The ionic liquid 1-ethyl-3-methylimidazolium tetrafluoroborate (EMI 
$\mathrm{BF}_{4}, \sigma=1.4 \mathrm{~S} / \mathrm{m}$ ) was also tested. Experiments using EG or $\mathrm{PC}$ were completed in air, whilst the $\mathrm{EMI}^{\mathrm{BF}_{4}}$ experiments were completed under vacuum.

Using the PC solution four geometric parameters were varied; the emitter inner and outer diameter, the emitter to extractor distance, and emitter length. Full geometric details are listed elsewhere. ${ }^{25}$ In order to achieve variation of emitter length, rather than using emitters of differing lengths resulting in the complication of changing the hydraulic impedance, the effective emitter length was varied by the insertion of the emitter through a large flat translatable Aluminum plate. For both the testing of EG and $\mathrm{EMI} \mathrm{BF}_{4}$, a $50 \mu \mathrm{m}$ outer diameter needle, $30 \mathrm{~mm}$ long situated $3 \mathrm{~mm}$ from the extractor was used throughout.

In each experiment, an initial flow rate through the emitter was established by a small gravitational pressure head from a reservoir. The voltage was increased in $30 \mathrm{~V}$ to $200 \mathrm{~V}$ steps (depending on the voltage range) from $0 \mathrm{~V}$ to the voltage needed for multi-jet mode to occur. Each voltage step was held for between $30 \mathrm{~s}$ and $2 \mathrm{~min}$, allowing sufficient sampling of flow rate at each voltage step. During the experiments, the liquid meniscus was visually inspected using a CCD camera with a microscope lens attachment. The current was measured by the impedance-corrected voltage drop across a $1 \mathrm{M} \Omega$ resistor connected from the emitter needle to ground.

The differing modes were identified from visual analysis using the camera and the current values; these current values changed considerably as mode changes occurred.

Figure 1 demonstrates the effect of the applied potential difference on the averaged flow rate (with standard deviation error bars) across all four electrospray modes investigated. The current is plotted in a continuous form.

Within enhanced dripping mode the flow rate demonstrated no change with applied voltage. It was noted that the droplets decreased in size with voltage, and that the emission frequency increased. The combination of these two effects results in no variation of flow rate. ${ }^{8,26}$

The onset of pulsation mode was confirmed by a measurable current, and a visual change in the meniscus shape. The flow meter sampling frequency of $2 \mathrm{~Hz}$ did not allow though for the analysis of the change in flow rate during a single pulse.

The trend line fitted to the average pulsation mode flow rate with voltage shown in Figure 1 demonstrates a linear

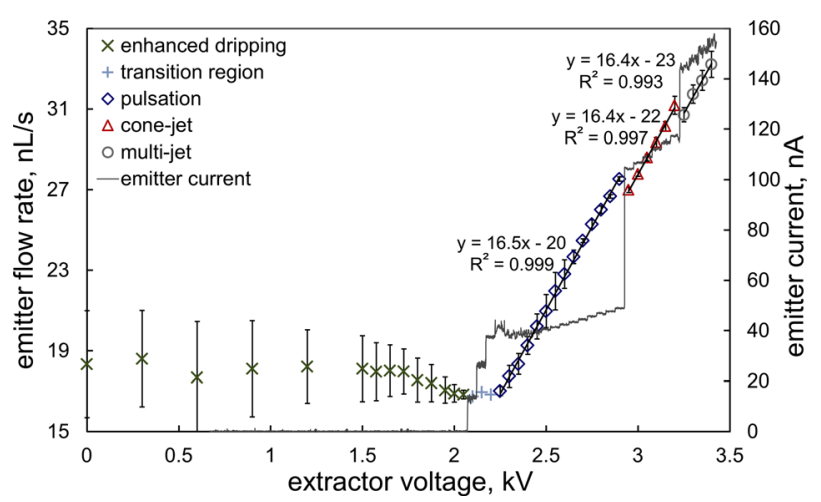

FIG. 1. Variation of flow rate with voltage across the four major regimes of electrospray. NaI doped PC, emitter i.d.-o.d. $=125-230 \mu \mathrm{m}$.
$Q(V)$ trend throughout the mode; this occurred consistently across the experiments undertaken.

In the transition from pulsation to cone-jet mode, a drop in flow rate can be seen. This consistently occurring drop in flow rate appears to be dependent on the initial flow rate and geometric parameters and is most likely related to space charge. ${ }^{27}$

Figure 1 illustrates that during cone-jet mode the flow rate again increased linearly with voltage, as found previously. ${ }^{22,24,25}$ At the highest voltages 2-cone multi-jet mode occurred, noticeable by another large current increase. The flow rate again decreased at this transition point, followed by a linear $Q(V)$ increase within multi-jet mode.

The gradient $\frac{d Q}{d V}$ of the three linear trend lines in Figure 1 is similar. This is a remarkable occurrence, as pulsation, multi-jet and cone-jet modes are electrohydrodynamically distinctive.

Figure 2 illustrates the variation of emitter flow rate with voltage in both pulsation and cone-jet modes for liquids $\mathrm{EMI} \mathrm{BF}_{4}$ and EG. For both liquids, the flow rate is approximately linearly dependent upon voltage both in pulsation and cone-jet modes, with, for a given fluid, similar gradients of the $Q(V)$ relationship in pulsation and cone-jet modes. For both liquids, a decrease in flow rate occurs in the transition from pulsation to cone-jet modes.

For NaI-doped PC, the average magnitude of the $\frac{d Q}{d V}$ gradient in pulsation, cone-jet and multi-jet modes as a function of the electrospray configuration are illustrated in Figure 3. In each of the panels, the data are plotted against a variation in system geometry. These geometric variations were previously investigated solely in cone-jet mode. ${ }^{24,25}$ An average gradient in each geometric condition is taken across at least 5 experiments with each gradient having a linear fitting of $R^{2}>0.98$, and standard deviation error bars calculated. The exception is for multi-jet results with emitters of lengths shorter than $5 \mathrm{~mm}$ (Figure 3(d)), where limited data were captured and individual multi-jet data are shown. The experiments investigating emitter tip to extractor distance sensitivity (Figure 3(c)) are shown for two values of emitter outer diameter. The $\frac{d Q}{d V}$ values from Figure 1 are included in Figure 3(c) within the averaged values for the $3 \mathrm{~mm}$ emitter-extractor distance configuration, using a $230 \mu \mathrm{m}$ outer diameter emitter.

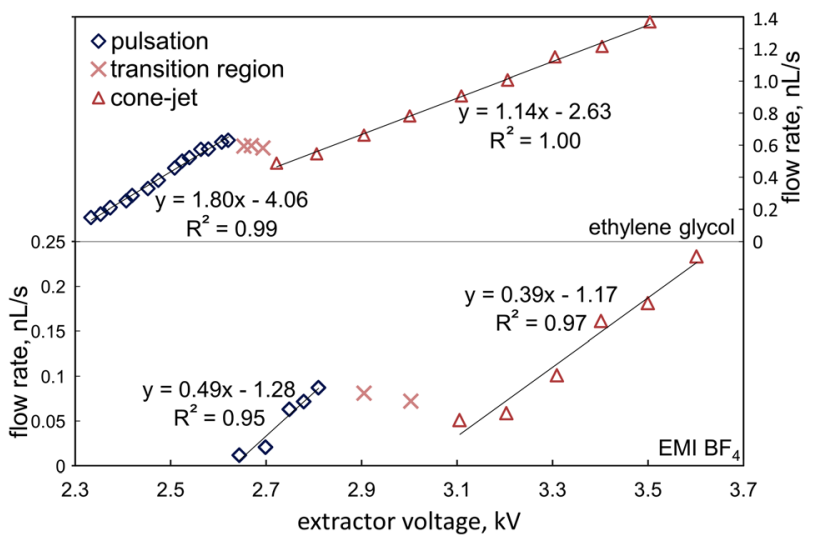

FIG. 2. Variation of flow rate across pulsation and cone-jet mode, for $\mathrm{NaI}$-doped EG and $\mathrm{EMI} \mathrm{BF}_{4}$. 

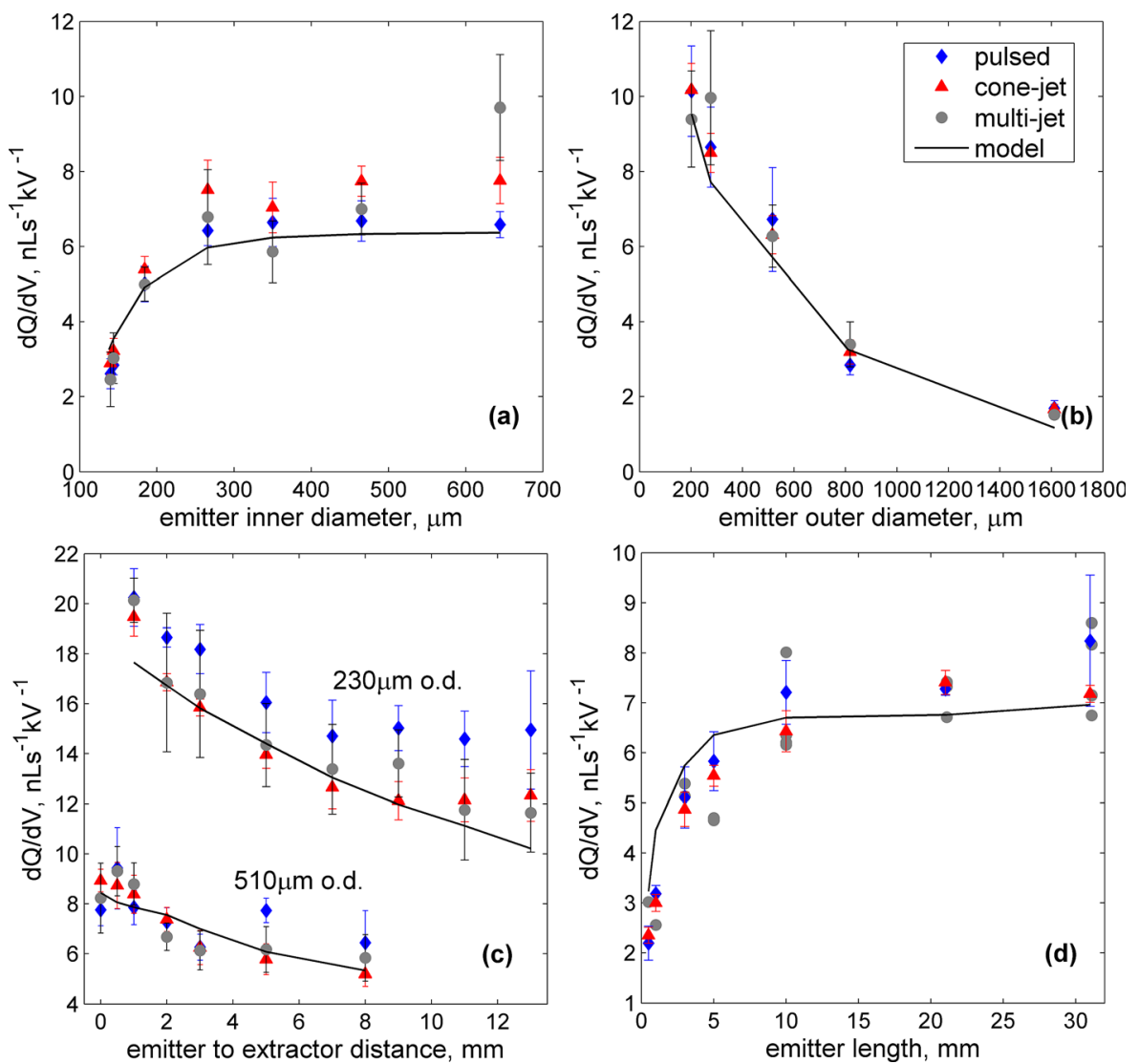

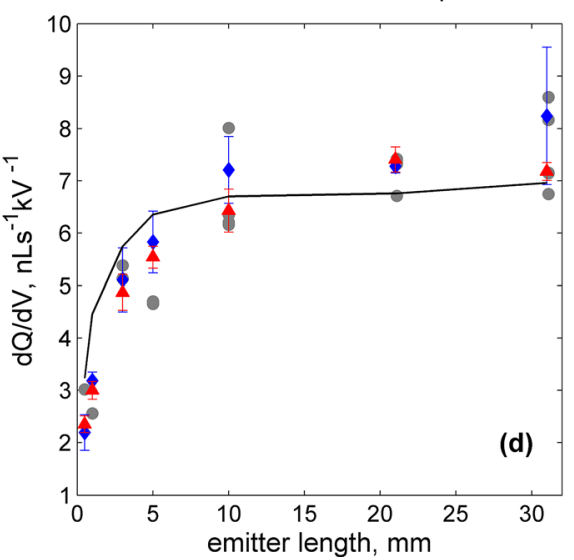

FIG. 3. (a)-(d) $\left\{\frac{d Q}{d V}\right\}$ variation with geometry within pulsation, cone-jet and multi-jet modes, for different geometry changes.
Also plotted in Figure 3 are simulated $\frac{d Q}{d V}$ results using a method applied previously to cone-jet mode results. ${ }^{25,27}$ The simulations are derived from the simplified electric stress (electric pressure) tensor $\mathrm{P}_{\mathrm{E}}$

$$
P_{E}=\frac{1}{2} \varepsilon_{0} E_{n}^{2},
$$

where $\varepsilon_{0}$ is the vacuum permittivity, and $E_{n}$ is the electric field normal to the meniscus surface. $E_{n}=\kappa V$, where $\kappa$ is a function relating electric field at the apex of the meniscus to the applied potential $V$. The flow rate $Q$ is related to the electric pressure by the hydraulic impedance $R_{T}$ of the fluid system

$$
Q=\frac{P_{E}}{R_{T}}
$$

Differentiating with respect to $V$ gives

$$
\frac{d Q}{d V}=\frac{1}{R_{T}} \frac{d p_{E}}{d V}=\frac{\varepsilon_{0} \kappa^{2} V}{R_{T}} .
$$

The experimental onset voltage of cone-jet mode for each geometric case $\varphi_{0}$ is substituted for the applied potential $V$. This onset voltage varied somewhat with geometry, with $\frac{d Q}{d V}$ a weak function of it. The coefficient $\kappa$ is calculated using an electrostatic finite element analysis (FEA) of the electrospray system geometry and the fluid meniscus, ${ }^{25}$ using a Bernstein-Bézier curve ${ }^{25,28}$ to model the fluid meniscus. As well as $\kappa$ being a function of the system geometry, it is dependent on the shape of the liquid meniscus curve, with the ratio of the meniscus tip radius $r_{a}$ to emitter radius $r_{e}$ defining the Bernstein-Bézier curve shape, varying from a Taylor-cone to a more hemispherical shape. A value for $\kappa$ with $r_{a} / r_{e}=1.40$ was found to best fit cone-jet mode data, ${ }^{25,27}$ and the same value is used here.

For the results illustrated in Figures 1 and 2, the value of $\frac{d Q}{d V}$ was calculated using Eq. (3) and the parameters listed in Table I. $\varepsilon_{0}$ is equal to $8.85 \times 10^{-12} \mathrm{~F} / \mathrm{m}$. The value of $\kappa$ is that calculated using the FEA model with a meniscus geometry set by choosing $r_{a} / r_{e}=1.40$. The voltage is taken to be the cone-jet onset voltage as illustrated in Figure 1 or Figure 2, whilst $R_{T}$ is that calculated using the Hagen-Poiseuille equation from the summation of the different pipe sections used including the emitter. The calculated values of $\frac{d Q}{d V}$ agree with the trends of the experimental values found in Figures 1 and 2. Note that for the results in Figure $2 \kappa$ is larger than in Figure 1 results as the outer diameter of the emitter used is smaller (50 compared to $230 \mu \mathrm{m}$ ), but since $R_{T}$ is greater $\frac{d Q}{d V}$ is smaller. For all three the $\frac{d Q}{d V}$ magnitude prediction is good, although with EMI $\mathrm{BF}_{4}$ the analytical value is approximately double the experimental value, plausibly due to the much higher conductivity of EMI $\mathrm{BF}_{4}$ resulting in greater space charge suppression of the flow rate sensitivity to voltage.

TABLE I. The calculation of the analytical $\frac{d Q}{d V}$ using Eq. (3) and experimental data shown Figures 1 and 2.

\begin{tabular}{lrccc}
\hline \hline Figure no. & $\kappa\left(\mathrm{m}^{-1}\right)$ & $V(\mathrm{~V})$ & $R_{T}\left(\mathrm{kgs}^{-1} \mathrm{~m}^{-4}\right)$ & $\frac{d Q}{d V}\left(\mathrm{nLs}^{-1} \mathrm{kV}^{-1}\right)$ \\
\hline $1(\mathrm{PC})$ & 4751.1 & 2950 & $3.80 \times 10^{13}$ & 15.52 \\
$2(\mathrm{EG})$ & 17570.0 & 2722 & $4.96 \times 10^{15}$ & 1.50 \\
$2\left(\mathrm{EMI} \mathrm{BF}_{4}\right)$ & 17570.0 & 3106 & $1.03 \times 10^{16}$ & 0.82 \\
\hline \hline
\end{tabular}


Figure 3 illustrates that $\left\{\frac{d Q}{d V}\right\}_{\text {pulsed }}$ and $\left\{\frac{d Q}{d V}\right\}_{\text {multi-jet }}$ exhibit the same trends and gradients with geometry as $\left\{\frac{d Q}{d V}\right\}_{\text {cone-jet }}$, and that results agree with an analytical model based on the electric pressure. The effect of voltage on flow rate can be explained by the same effect across all three modes. Changes in emitter-extractor geometry affect the strength of the relationship of electric field to voltage, $\kappa$. As an example the electric field, and hence pressure, increases at a greater rate with voltage as the emitter outer diameter reduces, and therefore acts to extract the fluid out of the emitter at a greater rate for a smaller outer diameter. This, combined with the effect of the hydraulic impedance in the case of emitter inner diameter, explains the $Q(V)$ relationship to geometry across the three modes.

Note that if Eqs. (1) and (2) are combined

$$
Q=\frac{1}{2 R_{T}} \varepsilon_{0}\left(\kappa \varphi_{0}\right)^{2},
$$

where $Q$ is a quadratic function of the applied voltage, rather than the seemingly linear relationship exhibited by the experimental data in Figures 1 and 2. The resolution of the experimental results is not though high enough to discern between a linear and polynomial fitting across the limited voltage range of each electrospray mode, and therefore linearity is assumed as a good first approximation of the results.

The variation of flow rate with applied voltage across enhanced dripping, pulsation, cone-jet and multi-jet modes has been illustrated. Within enhanced dripping, there appears to be no dependence of emitter flow rate with voltage. Across all other modes, there is an apparently linear increase of $Q$ with $V$. It would seem that the effect within these particular modes is the result of the same phenomenon: the increase of the electric pressure with voltage combined with the effect of hydraulic impedance. The previously adopted analytical-FEA approach used to predict the sensitivity of flow rate within cone-jet mode was applied to pulsation and multi-jet modes.

This increase of flow rate from the initial flow rate to the maximum point in multi-jet mode can, under some geometric cases, be large. Since the $Q(V)$ relationship is not sensitive to the initial flow rate, ${ }^{22,24}$ the electric field-driven flow rate will make up the largest proportion of the emitter flow rate when the initial flow rate and hydraulic impedance are both low.

This small initial flow rate, low hydraulic impedance, and no flow constraining components (e.g., syringe pumps) is a situation that is found in many electrospray systems, generally where the electrospray is induced by the applied voltage. Note that the findings are not directly applicable to electrospray systems that employ a syringe pump, as a pump can be described as having very high hydraulic impedance, suppressing the effect of applied voltage on flow rate.

Examples of electrospray techniques where these conditions are met include nano-electrospray mass spectrometry, ${ }^{29}$ EHD printing, ${ }^{2,30}$ and electrospray colloid thrusters. ${ }^{4,31}$
Electrospinning may also employ a method where the system is passively fed, ${ }^{32}$ with the creation of fibres solely resulting from the applied voltage. For these conditions, the use of voltage to control the flow rate is particularly pertinent, for example, over fibre dimensions in the case of electrospinning, and in the control of flow rate in the case of electrospray thrusters. The findings described here are likely applicable to these situations.

${ }^{1}$ J. B. Fenn, M. Mann, C. K. Meng, S. F. Wong, and C. M. Whitehouse, Science 246(4926), 64 (1989).

${ }^{2}$ M. D. Paine, M. S. Alexander, K. L. Smith, M. Wang, and J. P. W. Stark, J. Aerosol Sci. 38(3), 315 (2007).

${ }^{3}$ L. Dae-Young, Y.-S. Shin, P. Sung-Eun, T.-U. Yu, and H. Jungho, Appl. Phys. Lett. 90(8), 081905 (2007).

${ }^{4}$ R. Krpoun, K. L. Smith, J. P. W. Stark, and H. R. Shea, Appl. Phys. Lett. 94(16), 163502 (2009).

${ }^{5}$ P. Lozano and M. Martínez-Sánchez, J. Colloid Interface Sci. 282(2), 415 (2005).

${ }^{6}$ J. Doshi and D. H. Reneker, J. Electrost. 35(2-3), 151 (1995); N. Bhardwaj and S. C. Kundu, Biotechnol. Adv. 28(3), 325 (2010).

${ }^{7}$ P. K. Notz and O. A. Basaran, J. Colloid Interface Sci. 213(1), 218 (1999).

${ }^{8}$ X. Zhang and O. A. Basaran, J. Fluid Mech. 326, 239 (1996).

${ }^{9}$ M. Cloupeau and B. Prunet-Foch, J. Electrost. 25, 165 (1990).

${ }^{10}$ J. Zeleny, Phys. Rev. 10(1), 1 (1917).

${ }^{11}$ I. Marginean, L. Parvin, L. Heffernan, and A. Vertes, Anal. Chem. 76(14), 4202 (2004).

${ }^{12}$ G. Taylor, Proc. R. Soc. London, Ser. A 280(1382), 383 (1964).

${ }^{13}$ K. L. Smith, Ph.D. dissertation, Queen Mary University of London, 2005.

${ }^{14}$ M. Cloupeau and B. Prunet-Foch, J. Aerosol Sci. 25(6), 1021 (1994).

${ }^{15}$ C. N. Ryan, K. L. Smith, and J. P. W. Stark, J. Aerosol Sci. 51(0), 35 (2012).

${ }^{16}$ M.-H. Duby, W. Deng, K. Kim, T. Gomez, and A. Gomez, J. Aerosol Sci. 37(3), 306 (2006).

${ }^{17}$ Y. Lu, F. Zhou, W. Shui, L. Bian, Y. Guo, and P. Yang, Anal. Chem. 73(19), 4748 (2001).

${ }^{18}$ J. Wei, W. Shui, F. Zhou, Y. Lu, K. Chen, G. Xu, and P. Yang, Mass Spectrom. Rev. 21(3), 148 (2002).

${ }^{19}$ M. D. Paine, M. S. Alexander, and J. P. W. Stark, J. Colloid Interface Sci. 305(1), 111 (2007).

${ }^{20}$ J. Fernández de la Mora and I. G. Loscertales, J. Fluid Mech. 260, 155 (1994); A. M. Gañán-Calvo, J. Dávila, and A. Barrero, J. Aerosol Sci. 28(2), 249 (1997); J. Rosell, Ph.D. dissertation, Yale University, 1994.

${ }^{21}$ K. L. Smith, M. S. Alexander, and J. P. W. Stark, J. Appl. Phys. 99(6), 064909 (2006).

${ }^{22}$ K. L. Smith, M. S. Alexander, and J. P. W. Stark, Phys. Fluids 18(9), 092104 (2006).

${ }^{23}$ M. S. Alexander, K. L. Smith, M. D. Paine, and J. P. W. Stark, J. Propul. Power 23(5), 1042 (2007).

${ }^{24}$ C. N. Ryan, K. L. Smith, M. S. Alexander, and J. P. W. Stark, J. Phys. D: Appl. Phys. 42(15), 155504 (2009).

${ }^{25}$ C. N. Ryan, K. L. Smith, and J. P. W. Stark, J. Appl. Phys. 112(11), 114510 (2012).

${ }^{26}$ A. Speranza, M. Ghadiri, M. Newman, L. S. Osseo, and G. Ferrari, J. Electrost. 51-52, 494 (2001).

${ }^{27}$ C. N. Ryan, Ph.D. dissertation, Queen Mary University of London, 2011.

${ }^{28}$ R. Krpoun and H. R. Shea, J. Appl. Phys. 104(6), 064511 (2008).

${ }^{29}$ M. S. Wilm and M. Mann, Int. J. Mass Spectrom. Ion Processes 136(2-3), 167 (1994); G. T. T. Gibson, S. M. Mugo, and R. D. Oleschuk, Mass Spectrom. Rev. 28(6), 918 (2009); D. Eikel and J. Henion, Rapid Commun. Mass Spectrom. 25(16), 2345 (2011).

${ }^{30}$ D. Hongxu, L. Cheng, Y. Weiwei, B. Lojewski, A. Linan, and D. Weiwei, J. Microelectromech. Syst. 22(1), 1 (2013).

${ }^{31}$ D. G. Courtney, Q. H. Li, and P. Lozano, J. Phys. D: Appl. Phys. 45(48), 485203 (2012).

${ }^{32}$ S. Petrik and M. Maly, presented at the Materials Research Society Fall Meeting and Exhibit, Boston, MA, 2009; A. L. Yarin and E. Zussman, Polymer 45(9), 2977 (2004). 